\title{
Immunophenotypic characterization of acute lymphoblastic leukemia in a flowcytometry reference centre in Sri Lanka
}

\author{
Manujasri Wimalachandra ${ }^{1}$, Manurie Prabashika ${ }^{1}$, Medhavini Dissanayake $^{1}$, Roshan de Silva ${ }^{2}$, \\ Lallindra Gooneratne $e^{1,2}$
}

(Index words: acute lymphoblastic leukemia, acute leukaemia, immunophenotype)

\begin{abstract}
Background Acute lymphoblastic leukemia (ALL), is a biologically heterogeneous disease where diagnosis, treatment and prognosis is heavily dependent on the correct characterization of the immunophenotype of each case.

Objectives To describe the immunophenotypic and laboratory features of a cohort of Sri Lankan children and adults with ALL and to compare them with those reported in other series.

Methods Records of 229 patients who were suspected of having acute leukaemia and referred for flow cytometry to the Asiri Hospital Sri Lanka, between August 2009 and October 2013 were reviewed. Referrals were from across the country including the National Cancer Institute.

Results Sixty seven percent were children below 12 years of age. $80 \%$ had B-lymphoblastic leukaemia (BALL), 20\% T-lymphoblastic leukaemia (T-ALL). In children, the peak age of diagnosis was between 2-6 years in B-ALL (61\%) and 3-6 years (43.7\%) in T-ALL. Incidence was commoner in males in all age groups and subtypes except in the adult B ALL group where the incidence was equal. Ninety three percent of paediatric B-ALL and $67 \%$ adult B-ALL were CD10 positive common ALL. In T-ALL cytoplasmic expression of CD3 was $100 \%$. nTdT was the most commonly expressed immature marker, in B-ALL - 91\% and in T-ALL - 69\%. At least one myeloid antigen was present in $34 \%$ of B-ALL cases and $60 \%$ of T-ALL cases.

Conclusions This study represents the first immunophenotype based description of ALL in Sri Lanka. Age, sex distribution, ALL subtypes and the immunophenotypic profile of each subtype mirrored those of previous studies.
\end{abstract}

Ceylon Medical Journal 2020; 65: 23-27

DOI: http://doi.org/10.4038/cmj.v65i1-2.9133

\section{Introduction}

Acute lymphoblastic leukemia (ALL), a neoplasm of precursor cells committed to B or T cell lineage has a world wild incidence of 1-4.75/100000 persons per year [1,2]. Seventy five percent of cases occur in children less than 6 years of age, which makes it the most common form of leukaemia in children.

ALL is a biologically heterogeneous disease with clinical features, morphology, immunophenotype, cytogenetic and molecular genetic abnormalities having important implications in the diagnosis, classification, risk stratification, minimal residual disease analysis and prognosis. This was highlighted with the introduction of the WHO system of classification of haemopoetic neoplasms that replaced the purely cytomorphology and cytochemistry based French and British system [2]. Various subtypes of ALL and their different characteristics have shown to differ among geographic distribution, ethnicity, and socioeconomic status [1,3,4,7-10]. As of to date there is a lack of published data on ALL based on this classification in Sri Lanka.

The objective of this study was to describe the immunophenotypic and laboratory features of a cohort of Sri Lankan children and adults with acute lymphoblastic leukaemia and to compare them with those reported in other series.

\section{Methods}

Records of 229 consecutive patients who were diagnosed with ALL at the flow cytometry unit of the Asiri Hospital Sri Lanka, during an 18 month period were reviewed. Immunophenotyping by flow cytometry was performed on bone marrow aspirate or peripheral blood samples. Samples were collected in ethylenediaminetetra acetic acid (EDTA) containing tubes and analysed within 24 hours of collection. Immunophenotyping was done using a Becton Dickinson FACS Calibur flowcytometer. BD

${ }^{1}$ Faculty of Medicine, University of Colombo, Sri Lanka, ${ }^{2}$ Asiri Group of Hospitals, Sri Lanka.

Correspondence: MW, e-mail: <manuwimala@gmail.com>. Received 13 February 2019 and revised version 28 June 2020 accepted 02 July 2020.

This is an open-access article distributed under the terms of the Creative Commons Attribution License, which permits unrestricted use, distribution, and reproduction in any medium, provided the original author and source are credited. 
CellQuest Pro software was used for analysis. The reagents used in order to detect cluster of differentiation markers (CD) were selected in accordance with the guideline issued by the general haematology task force of the British committee for standards in haematology [3]. Immunophenotype of blasts was determined when at least $20 \%$ of the blasts expressed the marker at fluorescence intensity above cutoffs established using the corresponding type-matched control antibody.

\section{Results}

\section{The age and sex distribution at presentation for the B-ALL and T-ALL groups}

Out of the total 229 subjects, 157 (68.6\%) were children below 12 years of age and 72 (31.4\%) were adults. The age and sex distribution in B ALL and T ALL are shown in table 1.

Table 1. Age and sex distribution of patients

\begin{tabular}{llccccccc}
\hline Immunophenotype & \multicolumn{3}{c}{ B-ALL } & \multicolumn{3}{c}{ T-ALL } \\
\hline Gender & \multicolumn{3}{c}{ Female } & \multicolumn{2}{c}{ Male } & \multicolumn{2}{c}{ Female } & \multicolumn{2}{c}{ Male } \\
& No & $\%$ & No & $\%$ & No & $\%$ & No & $\%$ \\
\hline Adult & 22 & 9.6 & 21 & 9.2 & 7 & 3 & 22 & 9.6 \\
Paediatric & & & & & & & & \\
$<2$ yrs & 7 & 3 & 9 & 3.4 & 0 & 0 & 0 & 0 \\
$2-5$ yrs & 19 & 8.3 & 41 & 17.9 & 6 & 2.6 & 2 & 0.8 \\
$>5$ & 25 & 10.9 & 40 & 17.5 & 1 & 0.4 & 7 & 3 \\
\hline
\end{tabular}

${ }^{*}$ Percentages are out of total cases

The median age of presentation among children in the B-ALL group was 4.3 years (range $0.5-12$ ) and in the T-All group 5.5 years (range 1-11). In adults, the median age of presentation was, 27 years (range 13-78) in B All while in T ALL it was 25 (range 13-67).

\section{The prevalence of anaemia, thrombocytopenia and leukocytosis at presentation}

The prevalence of anaemia (Haemoglobin below $100 \mathrm{~g} / \mathrm{L}$ ), thrombocytopenia (Platelet count below $100 \times 10^{9} / \mathrm{L}$ and leucocytosis (Leucocyte count $>11 \times 10^{9} / 1$ ) is shown in table 2 .

Table 2. The prevalence of anaemia, thrombocytopenia and leukocytosis in different age groups

\begin{tabular}{lcccccccc}
\hline & \multicolumn{3}{c}{ B-ALL } & \multicolumn{3}{c}{ T- ALL } \\
& Adult & $\%$ & Paediatric & $\%$ & Adult & $\%$ & Paediatric & $\%$ \\
\hline Anaemia & $25 / 43$ & 58.1 & $93 / 123$ & 75.6 & $14 / 28$ & 50 & $6 / 12$ & 50 \\
Thrombocytopenia & $27 / 43$ & 62.8 & $45 / 127$ & 35.4 & $13 / 29$ & 44.8 & $3 / 15$ & 20 \\
Leukocytosis & $30 / 42$ & 71.4 & $103 / 128$ & 80.4 & $25 / 28$ & 89.2 & $12 / 16$ & 75 \\
\hline
\end{tabular}

In the B ALL group $21.4 \%$ of adult patients and $27.3 \%$ of paediatric patients presented with a total leucocyte of count $>100000 / \mu l .41 .37 \%$ of adults with T ALL and $25 \%$ of children with T ALL had WBC count $>100000 / \mu l$ at presentation.

\section{Immunophenotype}

The frequencies of expression of all cell surface and cytoplasmic CD markers are given in table 3 and 4. 
Table 3. The frequencies of positive immune markers in B-ALL

\begin{tabular}{lcc}
\hline Immune markers & Adult group & $\begin{array}{c}\text { Paediatric } \\
\text { group }\end{array}$ \\
\hline CD19 & $95.3 \%$ & $100.0 \%$ \\
CD20 & $27.9 \%$ & $17.7 \%$ \\
CD10 & $67.4 \%$ & $92.9 \%$ \\
CD79 a & $81.3 \%$ & $94.3 \%$ \\
CD34 & $58.1 \%$ & $69.5 \%$ \\
nTdT & $83.7 \%$ & $90.8 \%$ \\
CD34 or nTdT & $90.2 \%$ & $96.4 \%$ \\
CD13 & $20.9 \%$ & $12.1 \%$ \\
CD33 & $27.9 \%$ & $22.9 \%$ \\
CD 13 or 33 & $36.5 \%$ & $33.5 \%$ \\
\hline
\end{tabular}

Table 4. The frequencies of positive immune markers in T-ALL

\begin{tabular}{lcc}
\hline Immune markers & Adult group & $\begin{array}{c}\text { Paediatric } \\
\text { group }\end{array}$ \\
\hline Cyto CD3 & $100.0 \%$ & $100.0 \%$ \\
CD7 & $100.0 \%$ & $68.8 \%$ \\
CD5 & $72.4 \%$ & $62.5 \%$ \\
CD 2 & $81.3 \%$ & $43.8 \%$ \\
CD34 & $27.6 \%$ & $6.2 \%$ \\
nTdT & $69.0 \%$ & $68.8 \%$ \\
CD34 or nTdT & $75.9 \%$ & $68.8 \%$ \\
CD13 & $50.0 \%$ & $55.6 \%$ \\
CD33 & $34.5 \%$ & $18.8 \%$ \\
CD13 or 33 & $72.4 \%$ & $55.6 \%$ \\
\hline
\end{tabular}

CD4 and CD8 expression was checked in only 18 adult patients and 5 childhood cases. Out of them $47.82 \%$ of cases were negative for both CD4 and CD8. CD 4 and CD8 double positivity was seen in only $26.1 \%$ of cases. $30.4 \%$ cases showed either CD4 or CD8 positivity.

\section{Discussion}

ALL is predominantly a disease of childhood amounting to $80 \%$ of all childhood leukaemias. The incidence is higher in males and affects Caucasians more than Asian, African and Hispanic populations. Common presenting features include anemia, infections, bleeding and bone pain. Immunophenotyping is an essential tool in the classification of leukaemia as it provides information on the lineage and stage of maturation. It is also useful for identifying subtypes, monitoring of disease and for identifying markers of prognostic relevance. Additional data such as age, sex, presence of anaemia, thrombo- cytopenia and total white cell count at presentation have also been studied for their prognostic significance.

In our study both B and T-ALL were commoner in the paediatric group than in adults. B-ALL was more frequent than T-ALL independent of the age group analyzed. The geographic variation of ALL is well established $[1,4,5]$. This difference has been linked to ethnicity and socioeconomic status. Western data clearly shows a higher incidence of B-ALL over T-ALL. However many older studies done among Asian, African and middle eastern populations have shown a higher incidence of T-ALL [5-7]. Our data also show a higher incidence in the B-ALL category with frequencies similar to that reported in the Americas and Europe. However there is evidence to state that the geographic difference in incidence is more likely due to the socioeconomic status of the population than ethnicity. This was highlighted by two independent studies carried out in south and north India, that showed the change in trends from T-All to B-ALL with the changing socioeconomic status of a single concerned population $[5,6]$. Similar changes have been observed in other developing nations [4]. We feel our data also supports the same hypothesis as although still considered a low middle income country from south Asia, the Sri Lankan population reaps the benefits of a well organized health care system that has pushed many of its health parameters in par with developed countries.

The 2-5-year age category is considered the peak age of incidence among children with ALL. Similar to the incidence of ALL subtypes, the age of peak incidence has also been linked to the socio economic status of a population with the said peak occurring in populations with higher socioeconomic status. In this cohort, the peak age at diagnosis was between the ages 2-6 years accounting to $61 \%$ of B-ALL. The age at first diagnosis is considered a strong predictor of disease outcome [8]. Few cases, 8 (5.7\%), presented less than 1 year of age and, more than 10 years, 14 (9.8\%) both these categories are associated with an adverse prognosis. Peak age of presentation of T-ALL in childhood was 3-6 years with $43.7 \%$ of patients belonging to this group. Two (12.5\%) patients were less than 1 year and 2 (12.5\%) were $>10$ years.

Among the adult patients both T-ALL and B-ALL were common in the 13-26 age group amounting to $43 \%$ and $72.4 \%$ cases respectively. Few adult patients (one B-ALL and 6 T-ALL) were diagnosed after the age of 60 years, which is also associated with a poor prognosis [9]. The commonly described, bimodal distribution and "second lower peak" after 70 years of age was not observed in this cohort.

The incidence of ALL was more in males in all age groups and subtypes except in the adult B-ALL group where the incidence was almost equal (21 males to 22 female). Male sex is described as a risk factor for relapse of disease. [1,2,8,10]. 
Leucocytosis is a common presentation in paediatric and adult patients with ALL. We found that adult patients with T ALL had a higher mean WBC count at presentation compared to the paediatric group $(\mathrm{p}<0.0005)$. A relatively high prevalence of anaemia was seen with more than half the subjects in all age groups recording a hemoglobin of less than $100 \mathrm{~g} / \mathrm{l}$ at presentation.

In the immunophenotypic characterization, the most sensitive markers for diagnosis of B-ALL were CD19 and Cyto CD79 a with more than $90 \%$ of positivity. Ninety three percent of paediatric B ALL and $67 \%$ adult B ALL were CD10 positive common ALL. CD20 expression was seen in only $27.9 \%$ of adult B ALL cases and $17.7 \%$ of childhood B-ALL cases. These findings roughly mirror those previously presented in most other studies [2,9,10].

The expression of $\mathrm{T}$ cell associated antigens CD2, CD7, CD5 and CD3 shows high sensitivity and typifies at least $90 \%$ of TALL cases in both children and adults. Out of these CD3 is lineage specific and cytoplasmic expression was seen in all cases of T ALL. Surface CD3 expression was not checked in most of cases.

There was a relatively high prevalence in the immature markers in B ALL, with nTdT positive in $90.8 \%$ of children and $83.7 \%$ of adults. CD34 was positive in $69.5 \%$ of children and $58.1 \%$ of adults. $55.8 \%$ adults and $64.5 \%$ children with B-ALL showed both CD34 and nTdT positivity.

In the T-ALL group nTdT was positive in $69 \%$ of adult patients and $68.8 \%$ of childhood cases. CD34 expression was relatively low and found in $27.6 \%$ of adult cases and $6.2 \%$ of paediatric cases.

Classification of T-ALL according to maturation stages was difficult in our study as only a limited number of immune markers were analysed. Majority of the analysed cases show negativity for both CD4 and CD8 indicating their origin from more immature pro T or pre T stage. Similar results are reported in previous studies on T-ALL [2]. In contrast $\mathrm{T}$ lymphoblastic lymphoma, the tissue counterpart of T-ALL is derived from a more mature thymocyte counterpart as described in previous studies [2,12]. CD4 and CD8 double positivity seen in $26.1 \%$ of analysed cases indicated their origin from cortical T cell. Conventionally, development stage of the predominant clone is considered a predictor of outcome [2,8]. Cortical T-ALL carries the best prognosisand early stage T-ALL tend to be worse but once the treatment response is factored into risk alogorithms developmental stage is not considered an independent risk factor [8].

Aberrant expression of myeloid markers in ALL has been found to vary in different studies. And their value as progonostic markers is also a matter of debate $[9,13,14]$. Clinical outcome studies have shown conflicting data with regard to the prognostic significance of myeloid antigen positivity in terms of achievement of complete remission, response to conventional drug treatment, disease free survival and overall survival at 5 years [13,14]. The overall incidence of myeloid marker expression in B-ALL was comparable to what is described previously, but in most studies it was found that aberrant myeloid marker expression is more common in B-ALL than T-ALL $[10,13,14]$. However in our study aberrant myeloid antigen expression was more commonly seen in T-ALL cases. Our results indicate CD13 and CD33 to be the most likely myeloid markers to be expressed with atleast one of them present in 34\% of B-ALL cases and 60\% of T-ALL cases. Other myeloid antigens like MPO or CD117 were rarely seen. Multiple myeloid antigen expression was also rare.

A limitation in our study was that we were able to analyse only limited number of markers some markers of potential interest could not be fully evaluated and sub classification according to the antigen expression corresponding to the maturation stage was not possible.

Epidemiological data on ALL show that the incidence, age distribution, sex distribution and cytogenetic features among children can defer on country of origin, ethnicity, and level of socio-economic development. This highlights the importance of maintaining national registries of leukaemia patients for each population and using the data in planning and providing appropriate services in the diagnosis and treatment of leukaemia. We hope that the data reported in our study will serve as a reference for further research in haematological malignancies based on immunophenotyping in Sri Lanka.

\section{List of abbreviations}

ALL - acute lymphoblastic leukaemia

B-ALL - B-lymphoblastic leukaemia

T-ALL - T- lymphoblastic leukaemia

EDTA - ethylenediaminetetra acetic acid

CD - cluster of differentiation markers

\section{Conflicts of interest}

No conflicts of interest

\section{References}

1. Redaelli A, et al. A systematic literature review of the clinical and epidemiological burden of acute lymphoblastic leukaemia (ALL). Eur J Cancer Care 2005; 14: 53-62.

2. Swerdlow SH, Campo E, Harris NL, Jaffer ES, Pileri SA, Stein H, Vardiman, JW (Eds.). WHO Classification of Tumours of Haematopoietic and Lymphoid Tissues, Fourth Edition. Lyon, IARC Press. 2008.

3. Bain BJ, Barnett D, Linch D, Matutes E, Reilly JT. Revised guideline on immunophenotyping in acute leukaemias and chronic lymphoproliferative disorders. Clin Lab Haematol 2002; 24: 1-3.

4. Noronha EP, Marinho HT, Thomaz EB, Silva CA, Veras GL, Oliveira RA. Immunophenotypic characterization of acute leukemia at a public oncology reference center in 
Maranhão, northeastern Brazil. São Paulo Med J 2011; 129: 392-401.

5. Rajalekshmy KR, Abitha AR, Anuratha N, Sagar TG. Time trend in frequency of occurrence of major immunophenotypes in paediatric acute lymphoblastic leukemia cases as experienced by Cancer Institute, Chennai, south India during the period 1989-2009. Indian J Cancer 2011; 48: 310 .

6. Sazawal S, Bhargava M, Arya L, Khattar A, Saxena R. Acute lymphoblastic leukemia in children: Changing immunophenotypic pattern in Northern India. Indian J Med Paediatr Oncol. 2008; 29: 49.

7. Naeem S, Hayee A. Acute lymphoblasticleukaemia - a study of Immunophenotypes. J Pak Med Assoc. 1992; 42: 83-6.

8. Teachy D, Hunger S. Predicting relapse risk of acute lymphoblastic leukaemia. Brit J Haematol 2013; 162: 606-20.

9. Rowe JM. Prognostic factors in adult acute lymphoblastic leukaemia. Brit J Haematol 2010; 150: 389-405.

10. Hann IM, Richards SM, Eden OB, Hill FGH. Analysis of the immunophenotype of children treated on the Medical Research Council United Kingdom Acute Lymphoblastic
Leukaemia Trial XI (MRC UKALLXI). Leukemia 1998; 12: $1249-55$.

11. Salem DA, Abd El-Aziz SM. Flowcyto-metricimmunophenotypic profile of acute leukemia: mansoura experience. Indian J Hematol Blood Transfus 2012; 28: 89-96.

12. Jay L. Patel, Lynette M. Smith, James Anderson, Minnie Abromowitch, Dario Campana, Jeffery Jacobson, Mark A Lones, Thomas G. Gross, Mitchell S. Cairo, Sherrie L. Perkins. The immunophenotype of T-Lymphoblastic lymphoma in children and adolescents, Brit J Haematol 2012; 159: 454-61.

13. Vitale A, Guarini A, Ariola C, Meloni G, Perbellini O, Pizzuti M, De Gregoris C, Mettivier V, Pastorini A, Pizzolo G, Vignetti M. Absence of prognostic impact of CD13 and/or CD33 antigen expression in adult acute lymphoblastic leukemia. Results of the GIMEMA ALL 0496 trial. Haematologica 2007; 92: 342-8.

14. Bhushan B, Chauhan PS, Saluja S, Verma S, Mishra AK, Siddiqui S, Kapur S. Aberrant phenotypes in childhood and adult acute leukemia and its association with adverse prognostic factors and clinical outcome. Clinical and Experimental Medicine 2010; 10: 33-40. 